\title{
B-type supergiants in the Small Magellanic Cloud: rotational velocities and implications for evolutionary models
}

\author{
P. L. Dufton ${ }^{1}$, R. S. I. Ryans ${ }^{1}$, S. Simón-Díaz ${ }^{2,3}$, C. Trundle ${ }^{1,3}$, and D. J. Lennon ${ }^{2,3}$ \\ ${ }^{1}$ Department of Pure \& Applied Physics, The Queen's University of Belfast, BT7 1NN, Northern Ireland, UK \\ e-mail: p.dufton@qub.ac.uk \\ 2 Isaac Newton Group of Telescopes, Apartado de Correos 368, 38700 Santa Cruz de La Palma, Canary Islands, Spain \\ 3 Instituto de Astrofísica de Canarias, 38200 La Laguna, Tenerife, Spain
}

Received 28 November 2005 / Accepted 27 January 2006

\begin{abstract}
High-resolution spectra for 24 SMC and Galactic B-type supergiants have been analysed to estimate the contributions of both macroturbulence and rotation to the broadening of their metal lines. Two different methodologies are considered, viz. goodness-of-fit comparisons between observed and theoretical line profiles and identifying zeros in the Fourier transforms of the observed profiles. The advantages and limitations of the two methods are briefly discussed with the latter techniques being adopted for estimating projected rotational velocities $(v \sin i)$ but the former being used to estimate macroturbulent velocities. The projected rotational velocity estimates range from approximately 20 to $60 \mathrm{~km} \mathrm{~s} \mathrm{~s}^{-1}$ apart from one SMC supergiant, Sk 191, with a $v \sin i \simeq 90 \mathrm{~km} \mathrm{~s}^{-1}$. Apart from Sk 191, the distribution of projected rotational velocities as a function of spectral type are similar in both our Galactic and SMC samples with larger values being found at earlier spectral types. There is marginal evidence for the projected rotational velocities in the SMC being higher than those in the Galactic targets but any differences are only of the order of 5-10 $\mathrm{km} \mathrm{s}^{-1}$, whilst evolutionary models predict differences in this effective temperature range of typically 20 to $70 \mathrm{~km} \mathrm{~s}^{-1}$. The combined sample is consistent with a linear variation of projected rotational velocity with effective temperature, which would imply rotational velocities for supergiants of $70 \mathrm{~km} \mathrm{~s}^{-1}$ at an effective temperature of $28000 \mathrm{~K}$ (approximately B0 spectral type) decreasing to $32 \mathrm{~km} \mathrm{~s}^{-1}$ at $12000 \mathrm{~K}$ (B8 spectral type). For all targets, the macroturbulent broadening would appear to be consistent with a Gaussian distribution (although other distributions cannot be discounted) with an $\frac{1}{e}$ half-width varying from approximately $20 \mathrm{~km} \mathrm{~s}^{-1}$ at B8 to $60 \mathrm{~km} \mathrm{~s}^{-1}$ at B0 spectral types.
\end{abstract}

Key words. galaxies: Magellanic Clouds - stars: early-type - stars: supergiants

\section{Introduction}

Evolutionary models, such as those by Heger \& Langer (2000), Meynet \& Maeder (2000) and Maeder \& Meynet (2001), provide predictions of the evolution of both surface chemical compositions and rotational velocities of massive hot stars as a function of time. One common theme of these models is that an initially high rotational velocity (of the order of $300 \mathrm{~km} \mathrm{~s}^{-1}$ ) is required to produce significant mixing of nucleosynthetically processed material to the surface. Although rotational velocities decrease as their progenitor OB-type stars evolve away from the main sequence, B-type supergiants showing processed material at their surface are predicted to retain significant rotational velocities. However measurement of projected rotational velocities for a range of SMC early-type stars (O-type supergiants - Hillier et al. 2003; O-type dwarfs - Bouret et al. 2003; B-type giants and supergiants - Lennon et al. 2003; Trundle et al. 2004) appear to be systematically too low when compared with the predictions (see Fig. 7 of Trundle et al.). Additionally in most previous analyses, it has been assumed that the line broadening was predominantly due to rotation and if this were not the case the discrepancy between observation and theory would become larger.

Howarth et al. (1997) investigated the line widths in IUE spectra for a large sample of early-type evolved stars and noted that there were no narrow-lined objects. They interpreted this as evidence that there was another mechanism (hereafter designated as "macroturbulence"), which was contributing to the line broadening. More recently Ryans et al. (2002) have analysed high signal-to-noise $(\mathrm{S} / \mathrm{N}$ ) ratio (of the order of 500) spectra of Galactic supergiants in order to try and estimate the relative contributions of rotation and macroturbulence. They found that although the line widths implied projected rotational velocities in the range of 50 to $100 \mathrm{~km} \mathrm{~s}^{-1}$, their best estimates were typically in the range of 10 to $30 \mathrm{~km} \mathrm{~s}^{-1}$, with all upper limits being less than $60 \mathrm{~km} \mathrm{~s}^{-1}$. These results were lower than, for example, the calculations of Meynet \& Maeder (2000) for solar metallicity objects that 
predicted rotational velocities in the range of 50 to $200 \mathrm{~km} \mathrm{~s}^{-1}$. Even allowing for projection effects, the observed values appeared to be systematically too low.

Recently there have been two non-LTE analyses of high quality spectroscopy of SMC B-type supergiants. Trundle et al. (2004) analysed the spectra of 7 supergiants (and a giant) using the unified code FASTWIND (Santolaya-Rey et al. 1997) to determine their photospheric and wind parameters. Dufton et al. (2005) used the static code TLUSTY (Hubeny 1988; Hubeny \& Lanz 1995) to analyse 9 supergiants. Two of these targets were in common with those of Trundle et al. and a comparison of the two analyses showed excellent agreement. Here we analyse the spectroscopic data from these two SMC investigations using the techniques discussed in Ryans et al. (2002) and Simón-Díaz \& Herrero (2006) to estimate projected rotational and macroturbulent velocities. These techniques utilise different methodologies and hence allow us to investigate their strengths and limitations. We conclude that the Fourier transform technique of Simón-Díaz \& Herrero provides the better estimates for the projected rotational velocity and have hence used it to reanalyse the Galactic stellar data presented by Ryans et al. (2002).

Our principle aim is to obtain reliable estimates of the stellar projected rotational velocities while allowing for the contribution of other broadening mechanisms. In particular, we will investigate whether the low estimates found by Ryans et al. (2002) are confirmed and replicated in our SMC samples. One advantage of studying targets in the low metallicity environment of the SMC is that the presence and the amount of contamination of the stellar surface by nucleosynthetically processed material is far easier to characterise. This in turn makes it easier to search for any correlation between rotation and the amount of such contamination.

\section{Observational data}

Our observational material consists of high resolution spectroscopy for 13 SMC supergiants obtained at the AngloAustralian Telescope (AAT) and the ESO Very Large Telescope (VLT) and for 11 Galactic supergiants obtained at the McDonald Observatory and the William Herschel Telescope (WHT).

Trundle et al. (2004) obtained spectroscopy of 7 SMC targets at the VLT with the UVES instrument in November 2001. Of particular relevance here are the observations obtained in the blue arm of the spectrograph with a wavelength coverage of 3900 to $5000 \AA$. Trundle et al. rebinned their data to a pixel size of $0.2 \AA$ (yielding signal-to-noise ratios ranging from 120 to 190). Here we have used the unbinned spectra which have a spectral resolution, $R \sim 40000$. Two targets, AzV 104 (B0.5Ia) and AzV 216 (B1 III) have not been included in the current investigation - the former as the quality of the observational data was insufficient to accurately estimate the projected rotational velocity and the latter as it is not a supergiant. The VLT data are summarized in Table 2 and further details of the observations and the reduction techniques can be found in Trundle et al. (2004).
Spectroscopy for 9 SMC B-type supergiants (two in common with the VLT observations) was obtained at the Anglo-Australian Telescope in September 1996, with the UCLES instrument. There was complete wavelength coverage from 3900 to $4900 \AA$ with a pixel size of $\sim 0.08 \AA$, a spectral resolution, $R \sim 20000$ and with signal-to-noise ratios ranging from 90 to 130. Note that although the signal-to-noise ratios of the AAT data are lower than that of the binned VLT data, the differences in pixel size lead to the data being of similar quality (see, for example, Fig. 3 of Dufton et al. 2005). The stars observed are again summarized in Table 2 and further details of the reduction techniques can be found in Dufton et al. (2005).

Ryans et al. (2002) has presented spectroscopy for the Galactic supergiants with signal-to-noise ratios ranging from 250 to 700 and spectral resolutions of $R \sim 160000$ (McDonald) and $R \sim 40000$ (WHT). HD 36371 (B4 Iab) has been excluded from this investigation due to the difficulty of estimating the projected rotational velocity with the other targets being listed in Table 2. Full details of the observations and their reduction can be found in Ryans et al.

\section{Analysis and results}

\subsection{Analysis methodologies}

We have considered two methodologies to investigate the broadening of the absorption lines in our supergiant sample and in particular to distinguish between rotational broadening and that due to other mechanisms that we characterise as macroturbulence. These utilise the goodness-of-fit between observed and theoretical profiles and the shape of the Fourier transforms of observed absorption lines and are discussed in detail by Ryans et al. (2002) and Simón-Díaz \& Herrero (2006) respectively. Below we summarize the two methodologies.

The methodology of Ryans et al. is, in principle, straightforward. Equivalent widths of relatively strong, unblended lines in each star are measured, and then theoretical profiles of the same strength are generated using a non-LTE code (e.g. TLUSTY - Hubeny 1988) with appropriate atmospheric parameters. It is assumed that the broadening of the intrinsic absorption line profile is due to rotation $(v \sin i)$ and another mechanism that is characterised as macroturbulence and is initially assumed to have a Gaussian distribution of velocities (with a half-width at which the profile has fallen to $\frac{1}{e}$ of its maximum value given by $v_{\mathrm{m}}$ ). Theoretical profiles are then convolved with these functions and compared to the observational data. The sum of the squares of the differences are computed, giving a measure of the quality of the fit at each point in $\left(v_{\mathrm{m}}, v \sin i\right)$ space. The main difficulty with this goodness-of-fit (GOF) approach is that profiles generated with different $\left(v_{\mathrm{m}}, v \sin i\right)$ combinations are quite similar especially when one broadening mechanism dominates and we return to this point in Sect. 3.2.

By contrast the Fourier transform (FT) methodology discussed by Simón-Díaz \& Herrero (2006) allows the projected rotational velocity to be derived independently of any other broadening mechanism which may affect the line profile. A line profile can be considered to consist of the convolution of 
Table 1. Comparison of the estimates of the projected rotational velocities found using goodness-of-fit (GOF) and Fourier transform (FT) techniques. For both cases, the ranges of the estimates and their medians are listed. Also tabulated are the maximum possible values of $v \sin i$ taken directly from Ryans et al. together with the number (n) of lines considered in the current comparison.

\begin{tabular}{lrccccc}
\hline \hline Star & \multicolumn{3}{c}{ Goodness-of-fit } & \multicolumn{3}{c}{ Fourier Transform } \\
& Range & Median & Max. & Range & Median & \\
\hline HD 2905 & $1-22$ & 20 & $\leq 60$ & $51-59$ & 52 & 4 \\
HD 13854 & $11-15$ & 13 & $\leq 40$ & $31-34$ & 32 & 3 \\
HD 21291 & $5-8$ & 7 & $\leq 20$ & $16-18$ & 17 & 2 \\
HD 24398 & $4-19$ & 9 & $\leq 40$ & $34-39$ & 36 & 4 \\
HD 34085 & $4-15$ & 10 & $\leq 30$ & $20-26$ & 25 & 6 \\
HD 38771 & $5-21$ & 9 & $\leq 50$ & $46-55$ & 50 & 4 \\
HD 41117 & $3-24$ & 14 & $\leq 40$ & $40-42$ & 41 & 2 \\
HD 193183 & $2-47$ & 20 & $\leq 50$ & $40-50$ & 45 & 11 \\
HD 204172 & $3-71$ & 57 & $\leq 60$ & $57-70$ & 62 & 8 \\
HD 206165 & $3-41$ & 11 & $\leq 40$ & $25-41$ & 35 & 7 \\
HD 208501 & $9-11$ & 11 & $\leq 30$ & $25-30$ & 28 & 4 \\
\hline
\end{tabular}

natural, instrumental, rotational and macroturbulent broadening profiles. As first discussed by Carroll $(1933 a, b)$ the Fourier transform of the rotational profile has zeros whose position in frequency space depend on the projected rotational velocity. The FT method for the determination of $v \sin i$ (cf. Gray 1973) is then based on the fact that in Fourier space, convolutions transform into products. Hence it is possible to determine the projected rotational velocity of a star once the position of the first of those zeroes is identified in the Fourier transform of the line profile. Although this methodology has not been widely used in the study of OB-type stars (e.g. Ebbets 1979), the recent study by Simón-Díaz \& Herrero (2006) illustrates the strength of this method for determining projected rotational velocities in early type stars (see also Simón-Díaz et al. 2006).

\subsection{Comparison of methodologies}

We have used the high quality spectroscopy reported in Ryans et al. (2002) to compare the results obtained by the two methodologies. We note that the FT approach does not directly provide estimates of the macroturbulence parameter $\left(v_{\mathrm{m}}\right)$ and hence the comparison is restricted to the estimates of the projected rotational velocities. In Table 1, we summarize the estimates from the two methodologies with the goodness-of-fit results being taken directly from Ryans et al. Note that for some lines, it was not possible to identify the zeros in the Fourier transforms and these cases have been excluded from the comparison. Consequently the GOF results listed in Table 1 differ slightly from those reported by Ryans et al. The only significant change is for HD 204172 where the exclusion of the $\mathrm{Mg}$ II line at $4481 \AA$ and the Si III line at $4552 \AA$ leads to an increase in the median from the GOF estimates.

Inspection of Table 1 implies that the estimates from the GOF technique are systematically lower than those from the FT technique with the mean of the differences being $22 \pm$ $10 \mathrm{~km} \mathrm{~s}^{-1}$. However the FT estimates are consistent with the maximum values deduced from the GOF analysis. We believe that these differences reflect the difficulty in identifying and quantifying the relatively small amount of rotational broadening in the presence of significant macroturbulent broadening.

With the GOF methodology, the profiles become effectively degenerate for small values of $v \sin i$ with the contour maps in the $\left(v_{\mathrm{m}}, v \sin i\right)$ plane having broad flat ridges along the $v \sin i$ axis (see, for example Fig. 3 of Ryans et al. 2002). Hence estimates of the projected rotational velocities become sensitive to the observational uncertainties and the assumptions adopted. For example, a Gaussian velocity distribution has been assumed for the macroturbulence and if the distribution differed from this, it could lead to systematic errors in the estimation of the projected rotational velocity. Additionally the sensitivity of the GOF approach to uncertainties in the observational data (such as blending and continuum normalisation) may be the cause of the wider range of estimates found in a given star compared with the FT approach.

For slowly rotating stars, the Fourier transform of the broadening function will have its first (and subsequent zeros) at high frequencies. Additionally if the observed stellar absorption lines are significantly broadened by macroturbulence most of their power will occur at low frequencies in the Fourier domain. Since the noise in the spectrum transforms as a "white noise" in the Fourier space (Smith \& Gray 1976), then the unambiguous identification of the zeros in the Fourier transform becomes difficult or impossible when the $v \sin i$ is low and the effect of macroturbulence is important (this is analogous to the difficulty in the GOF approach of estimating projected rotational velocities when the line profiles are dominated by macroturbulence). Indeed even for the high quality Galactic supergiant spectra it was not possible to estimate the projected rotational velocity from the Fourier transform for some spectral features in a given target. Tests undertaken by Simón-Díaz \& Herrero (2006) for cases with small projected rotational velocities and significant macroturbulence indicate that the former can be overestimated by 5 to $10 \mathrm{~km} \mathrm{~s}^{-1}$. Although this would reduce the discrepancy with the estimates deduced from the GOF methodology, it would not eliminate it.

However the advantage of this methodology is that if the zeros in the Fourier Transform can be unambiguously identified the corresponding $v \sin i$ estimates should be robust and in particular they are less dependent (compared with the GOF approach) on additional assumptions or on uncertainties in the observational data. Indeed indirect evidence for this is the smaller range in estimates found from different lines in a given star using the FT methodology.

\subsection{Estimates of the projected rotational velocity and macroturbulence}

Given the discussion of the previous section, we concluded that the FT methodology provided the better estimates of the projected rotational velocities and adopted this approach for both our Galactic and SMC samples. These estimates are summarized in Table 2 together with spectral types and estimates of the stellar effective temperature. For the projected rotational velocities we have quoted medians as these will be less affected 
Table 2. Median values for the macroturbulent $\left(v_{\mathrm{m}}\right)$ and projected rotational $(v \sin i)$ velocities deduced for the SMC datasets presented in Dufton et al. (2005) and by Trundle et al. (2004). Also listed are estimates for the Galactic supergiants discussed by Ryans et al. (2002). The sources of the spectral types and effective temperatures are discussed in the text.

\begin{tabular}{|c|c|c|c|c|c|}
\hline$\overline{\overline{\text { Star }}}$ & $\begin{array}{l}\text { Spectral } \\
\text { type }\end{array}$ & $\begin{array}{l}T_{\text {eff }} \\
\mathrm{K} \\
\end{array}$ & $\overline{\overline{\text { Dataset }}}$ & $\begin{array}{c}v \sin i \\
\mathrm{~km} \mathrm{~s}^{-}\end{array}$ & $v_{1} v_{\mathrm{m}}$ \\
\hline \multicolumn{6}{|l|}{ SMC } \\
\hline AzV 487 & BCOIa & 27000 & AAT & 50 & 54 \\
\hline AzV 215 & BNOIa & 26750 & AAT/VLT & 66 & 55 \\
\hline AzV 242 & B1Ia & 21500 & AAT & 54 & 43 \\
\hline AzV 78 & $\mathrm{~B} 1.5 \mathrm{Ia}^{+}$ & 21250 & AAT & 46 & 36 \\
\hline Sk 191 & B1.5Ia & 21500 & VLT/AAT & 92 & 49 \\
\hline $\mathrm{AzV} 210$ & B1.5Ia & 20500 & VLT & 37 & 36 \\
\hline $\mathrm{AzV} 462$ & B1.5Ia & 19000 & AAT & 43 & 26 \\
\hline AzV 303 & B1.5Iab & 18000 & AAT & 42 & 26 \\
\hline $\mathrm{AzV} 472$ & B2Ia & 19000 & AAT & 33 & 27 \\
\hline AzV 18 & B2Ia & 19000 & VLT & 33 & 30 \\
\hline AzV 374 & B2Ib & 18500 & AAT & 47 & 20 \\
\hline AzV 362 & B3Ia & 14000 & VLT & 38 & 30 \\
\hline AzV 22 & B5Ia & 14500 & VLT & 33 & 23 \\
\hline \multicolumn{6}{|l|}{ Galactic } \\
\hline HD 204172 & B0.2 Ia & 28500 & WHT & 62 & 62 \\
\hline HD 38771 & B0.5 Ia & 27500 & McDonald & 50 & 55 \\
\hline HD 2905 & BC0.7 Ia & 24000 & McDonald & 52 & 59 \\
\hline HD 13854 & B1 Iab & 23500 & McDonald & 32 & 53 \\
\hline HD 24398 & B1 Ib & 23000 & McDonald & 36 & 38 \\
\hline HD 193183 & B $1.5 \mathrm{Ib}$ & 22500 & WHT & 45 & 40 \\
\hline HD 41117 & B2 Ia & 19500 & McDonald & 41 & 38 \\
\hline HD 206165 & B2 Ib & 20000 & WHT & 35 & 34 \\
\hline HD 34085 & B8 Ia & 13000 & McDonald & 25 & 28 \\
\hline HD 208501 & B8 Ib & 13000 & WHT & 28 & 27 \\
\hline HD 21291 & B9 Ia & 11500 & McDonald & 17 & 21 \\
\hline
\end{tabular}

by any spurious measurements although very similar values would have been found if we had adopted the means of the estimates. We also undertook an analysis of the SMC dataset using the GOF methodology and will discuss this briefly in Sect. 4. Spectral types and effective temperatures were taken from McErlean et al. (1999) for the Galactic sample and Trundle et al. (2004) and Dufton et al. (2005) for the SMC targets. Note that the three studies utilised different non-LTE codes and different physical assumptions about e.g. the stellar winds and hence there may be systematic differences between the effective temperature scales. For two SMC targets, effective temperature estimates are available from both Trundle et al. and Dufton et al. and they differ by $500 \mathrm{~K}$ and $2000 \mathrm{~K}$ (in Table 2 we adopt the means of these values), which may be indicative of the magnitude of any systematic differences.

The FT technique does not provide a direct estimate of the magnitude of other broadening mechanisms (that have here been characterised as macroturbulence) although this will affect the overall shape of the Fourier transform. Hence we have used the GOF technique to estimate this quantity. Our approach was for each star to adopt the projected rotational velocity estimate listed in Table 2 and then to re-analyse the relevant

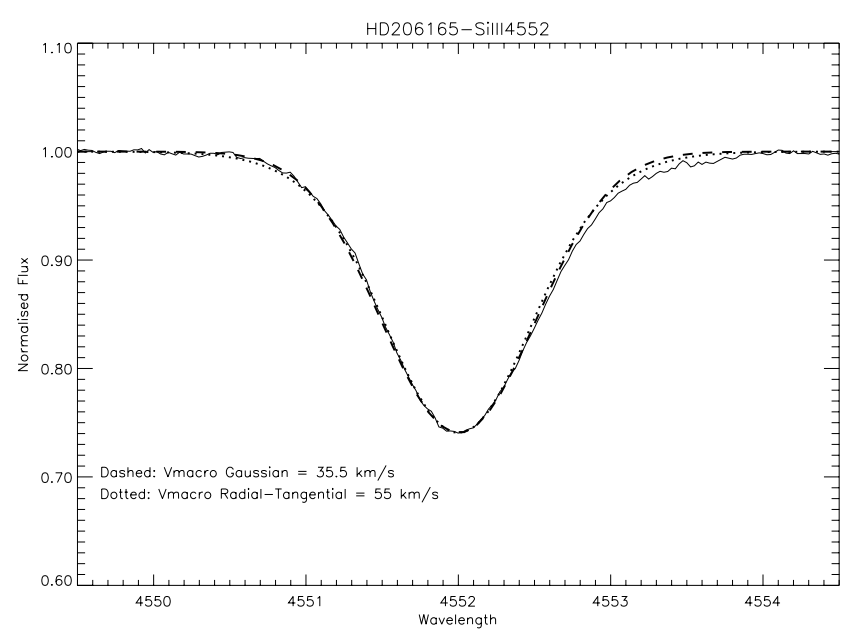

Fig. 1. Observed and theoretical profiles for the Si III line at $4552 \AA$ in HD 206165. The former is represented by a histogram whilst the theoretical profiles assume an isotropic Gaussian velocity (dashed line) and a radial-tangential model with $A_{\mathrm{r}}=1.0$ (dotted line). Note that both theoretical approaches lead to a good fit with observation particularly in the core of the line, however the radially tangential profile requires a larger macroturblent velocity.

lines to determine the macroturbulence $\left(v_{\mathrm{m}}\right)$ that best fitted the profile. These estimates are also listed in Table 2 where we have again adopted the median of the individual estimates.

The uncertainties in our estimates of the projected rotational velocity and macroturbulence will depend on several factors including the quality of the observational data and the magnitude of the quantity being estimated - for example, an estimate of a small macroturbulence in a target with significant rotational broadening will have a relatively high degree of uncertainty. In Table 1, we list the range of $v \sin i$ estimates for each of our Galactic targets with a similar spread being found for our SMC targets. These translate into a typical uncertainty in the median value of the $v \sin i$ estimates of $10 \%$. The spread in the estimates of the macroturbulent velocity for a given target is similar and implies a typical uncertainty in the medians of $10 \%$, apart from the smallest values $\left(v_{\mathrm{m}} \lesssim 30 \mathrm{~km} \mathrm{~s}^{-1}\right)$ where the uncertainty may be larger.

\section{Discussion}

\subsection{Macroturbulence}

The macroturbulence has initially been assumed to be isotropic and to follow a Gaussian distribution. The good agreement between the theoretical and observed profiles (see Fig. 1 of Ryans et al. 2002) provides some evidence in support of this assumption. However we cannot rule out other distributions and in particular those that are qualitatively similar to a Gaussian distribution. As an example we consider anisotropic macroturbulence characterised by a radial-tangential model (Gray $1975,1992)$. In this model the velocity field is modelled by assuming that a fraction $\left(A_{\mathrm{r}}\right)$ of the material is moving radially (but with a Gaussian distribution of velocities) with the remainder moving tangentially. The methodology was developed to model the flow of convective cells observed in the Sun. Hence 
it may not be appropriate to the targets considered here and should be considered as illustrative. As an example we have fitted the Si III line at $4552 \AA$ in HD 206165 adopting a radialtangential model with $A_{\mathrm{r}}=1.0$ and $\Theta_{\mathrm{r}}=55 \mathrm{~km} \mathrm{~s}^{-1}$ (i.e. assuming a complete radial flow). However we note that assuming that both the radial and tangential velocities are equal $\left(A_{\mathrm{r}}=A_{\mathrm{t}}\right.$, $\Theta_{\mathrm{r}}=\Theta_{\mathrm{t}}=55 \mathrm{~km} \mathrm{~s}^{-1}$ ) would have yielded an effectively identical profile. In addition, we have also calculated a profile for an isotropic macroturbulence with $v_{\mathrm{m}}=35.5 \mathrm{~km} \mathrm{~s}^{-1}$. The results are shown in Fig. 1 and both approaches are in good agreement with the observational data, but the radial tangential profile requires a larger macroturbulent velocity to be invoked (by approximately $20 \mathrm{~km} \mathrm{~s}^{-1}$ ). Hence we conclude that it is not possible with the current dataset to investigate the exact form or degree of the anisotropy of the macroturbulence. However what is clear is that additional broadening is present, which is consistent with a velocity distribution that is approximately Gaussian.

The finite spectral resolution of our observational data should not affect our estimates of the projected rotational velocity but could led to overestimates for the macroturbulence. Our lowest resolution spectroscopy $(R \sim 20000)$ would have a $\frac{1}{e}$ width, $v_{\mathrm{i}} \sim 6 \mathrm{~km} \mathrm{~s}^{-1}$ assuming that the instrumental profile was a Gaussian. Then if the macroturbulent velocity field also followed a Gaussian distribution, the estimates listed in Table 2 would represent the instrumental profile and the actual macroturbulent velocity added in quadrature. As an example, correcting our lowest estimate of $v_{\mathrm{m}} \sim 21 \mathrm{~km} \mathrm{~s}^{-1}$ for such an instrumental profile would lead to a decrease of less than $1 \mathrm{~km} \mathrm{~s}^{-1}$. For larger macroturbulent velocity estimates or better spectral resolutions, the corrections will be smaller and hence we have not attempted to apply them.

Inspection of results summarized in Table 2 implies that the amount of macroturbulent broadening in the Galactic and SMC supergiants are comparable. To illustrate this in Fig. 2, our best estimates of the macroturbulent velocities, $v_{\mathrm{m}}$, are plotted against the effective temperature for both sets of supergiants. As discussed in Sect. 3.3, the latter have been taken from different sources and although there may be systematic differences between these scales, we believe that this should not invalidate the comparison.

In general, both samples show a similar trend of macroturbulence with effective temperature and would imply that this is an intrinsic property of the star. Additionally as discussed previously the effect of this broadening is significant and invalidates the use of line widths as a measure of rotational broadening for B-type supergiants. This is consistent with the analysis of IUE spectra by Howarth et al. (1997), who found significant spectral broadening in all their supergiant spectra. This they interpreted as "confirming that an important line broadening mechanism in addition to rotation must be present in these objects".

For one SMC target, Sk 191, a relatively large projected rotational velocity is found from the FT methodology and this was confirmed by an independent GOF analysis. As can be seen from Fig. 2, our estimate of its macroturbulent velocity is compatible with the other targets having similar effective temperatures. By contrast adopting a small rotational velocity would
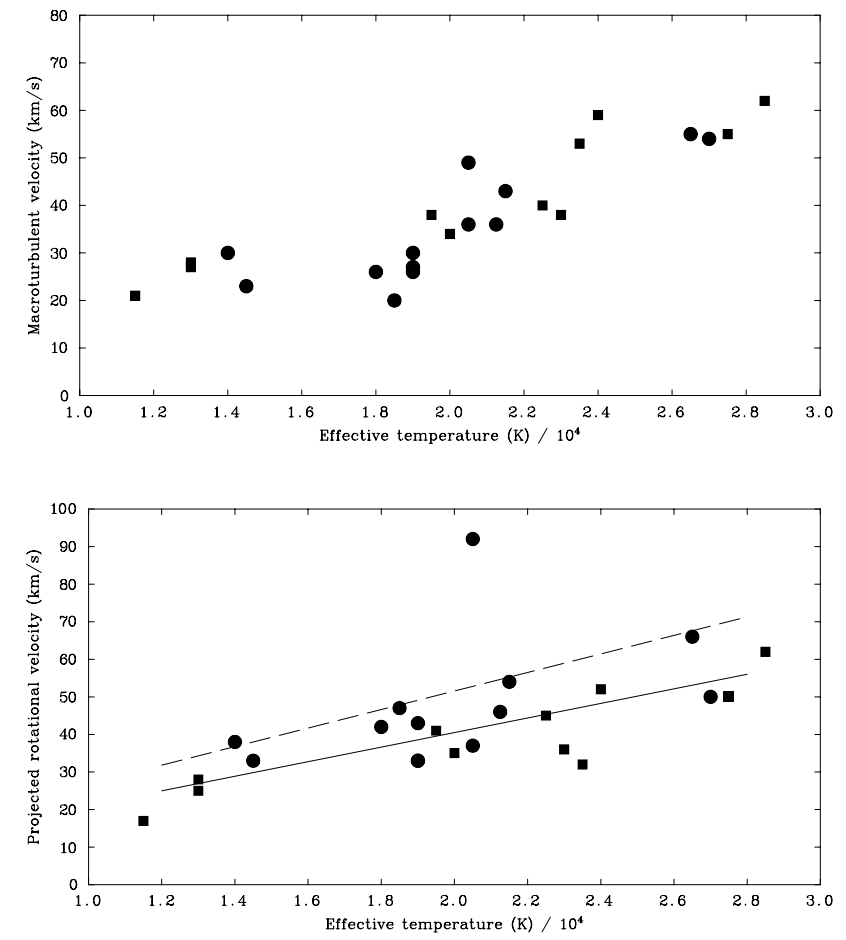

Fig. 2. Estimates of macroturbulent (upper figure) and projected rotational velocities (lower figure) plotted against effective temperature. The Galactic supergiants are represented by squares and the SMC targets by circles. For the projected rotational velocities, the linear least squares fit (excluding Sk 191) is shown as a solid line, whilst the dashed line would represent the rotational velocity assuming a random distribution of projection angles.

have led to an anomalously large estimate for the macroturbulence of approximately $80 \mathrm{~km} \mathrm{~s}^{-1}$.

If the additional spectra line broadening is due to macroturbulence, the estimated velocities, $v_{\mathrm{m}}$, are large and imply a highly dynamic atmosphere. Additionally B-type supergiants are also found to require significant microturbulent velocities that need to be incorporated into the profiles of the line absorption coefficient, with typically values being between 10 and $20 \mathrm{~km} \mathrm{~s}^{-1}$ (see, Crowther et al. 2005 and references therein). If these represent small scale turbulence, then they and the values of macroturbulence found here imply that the photospheres of B-type supergiants are highly turbulent on all distance scales. In turn the adoption of a stable photosphere in both static and unified stellar atmosphere codes must be considered problematic although excellent agreement between theory and observation has been found using such methods (Crowther et al. 2005; Trundle et al. 2004; Dufton et al. 2005).

Assuming that our macroturbulent velocities are due to a large scale velocity field, we can estimate its kinetic energy. As an example we consider three relatively hot targets, HD 38771, AzV 215 and AzV 487 with atmospheric parameters, $T_{\text {eff }} \simeq 27000 \mathrm{~K}$ and $\log g \simeq 2.90$. The evolutionary models of Meynet \& Maeder $(2000,2001)$ then imply an evolutionary mass of approximately $40 M_{\odot}$. Additionally, the corresponding TLUSTY model atmosphere implies that the mass of a column of unit area down to a Rosseland mean opacity of unity 
is $10 \mathrm{~kg} \mathrm{~m}^{-2}$. Adopting this as the extent of the atmosphere sampled by the absorption lines implies a total atmospheric mass of $6 \times 10^{19} \mathrm{~kg}$ and a typical macroturbulent velocity of $60 \mathrm{~km} \mathrm{~s}^{-1}$ would then lead to a kinetic energy in the velocity field of approximately $10^{29} \mathrm{~J}$. It should be noted that this is only an order of magnitude estimate as it incorporates several assumptions and simplifications. However the bolometric luminosity of these supergiants is approximately $6 \times 10^{5} L_{\odot}$ or $2 \times 10^{32} \mathrm{~J} \mathrm{~s}^{-1}$ and the kinetic energy of the macroturbulence would be therefore negligible when considering the energy balance in the atmosphere. McErlean et al. (1998) discuss the thermal velocities and sound speed for a model with atmospheric parameters ( $T_{\text {eff }}=27500 \mathrm{~K}, \log g=3.0$ and a microturbulence of $12 \mathrm{~km} \mathrm{~s}^{-1}$ ) similar to those of the three targets considered here. For hydrogen the thermal velocity is typically $20 \mathrm{~km} \mathrm{~s}^{-1}$ and the sound speed is $15-20 \mathrm{~km} \mathrm{~s}^{-1}$. Hence the energy of a macroturbulent velocity field would be greater than the thermal energy of the plasma, whilst its velocity would be supersonic. Assuming that this additional broadening is indeed due to a large scale velocity field, its origin and nature remain unclear.

\subsection{Projected rotational velocities}

The estimates of the projected rotational velocities, summarized in Table 2, imply that the values for both the Galactic and SMC samples are similar and this is supported by Fig. 2 where the estimates are plotted against effective temperature. If all our targets had the same initial mass and rotational velocity, the effective temperature would represent their age and evolutionary status. From a comparison with the evolutionary models of Schaller et al. (1992) for the Galactic targets (see McErlean et al. 1999, for further details) and Maeder \& Meynet (2001) for the SMC targets (see Trundle et al. 2004), we have estimated evolutionary masses. These fall in the range 20 to $40 M_{\odot}$, apart from the SMC target, AzV 78, which has an estimated initial mass of approximately $50 M_{\odot}$. This significant range in masses coupled with the initial rotational velocity of our targets being effectively unknown implies that this figure should be considered as primarily an empirical representation.

Theoretical predictions imply that the SMC supergiants should have larger rotational velocities (see Fig. 3 and discussion in Sect. 4.3) and there is some marginal evidence for this in Fig. 2. To investigate this we have independently estimated linear least squares fits for the two samples (excluding the anomalous SMC supergiant Sk 191 which is discussed below). The two fits are similar, differing by $4 \mathrm{~km} \mathrm{~s}^{-1}$ at $T_{\text {eff }}=$ $12000 \mathrm{~K}$ and $9 \mathrm{~km} \mathrm{~s}^{-1}$ at $28000 \mathrm{~K}$. Given the small size of the samples and the possibilities of systematic differences in the effective temperature scales of the two samples these differences cannot be considered significant. Hence we have also combined the two samples and the corresponding linear fit is shown in Fig. 2 as a solid line. The estimates of the projected rotational velocity will be affected by the unknown angle of inclination $(i)$. Assuming that this was randomly orientated would lead to an average value of $\sin i$ of $\frac{\pi}{4}$ and the dashed line in the figure represents the least squares fit increased by

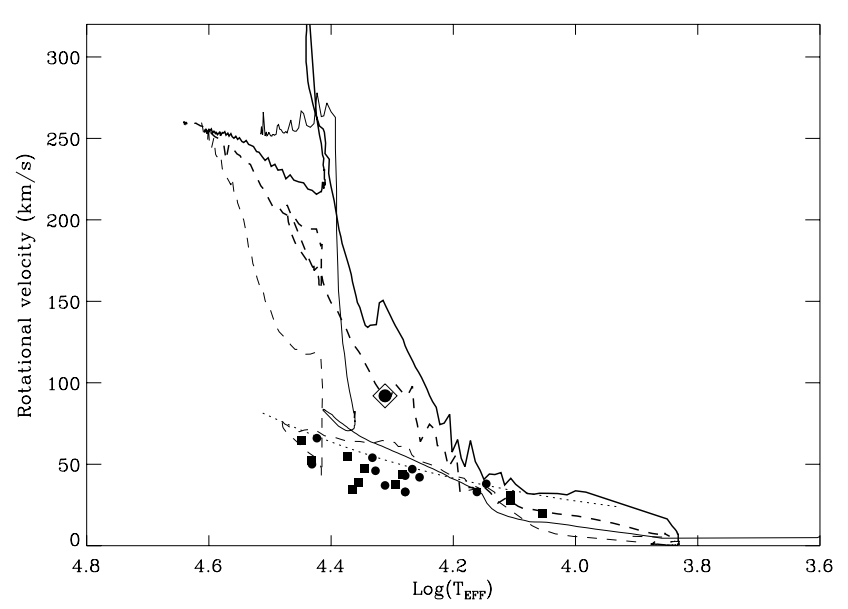

Fig. 3. Predictions of the evolution of the rotational velocity of two models with metallicities appropriate to our Galaxy (Meynet \& Maeder 2003; initial masses: $20 M_{\odot}$ - narrow solid line, $40 M_{\odot}$ - narrow dashed line) and two models representative of the SMC (Meynet \& Maeder 2004; $30 M_{\odot}$ - thick solid line, $40 M_{\odot}$ - thick dashed line). All models had an initial rotational velocity of $300 \mathrm{~km} \mathrm{~s}^{-1}$. Also shown are the estimated projected rotational velocities for our Galactic (filled squares) and SMC (filled circles) samples. The SMC target Sk 191 is represented by a filled circle enclosed in a diamond, for clarity. The dotted line represents our estimated mean rotational velocity for both samples, excluding Sk 191 and corrected assuming a random inclination angle.

the reciprocal of this factor. Although the sample is small it is encouraging that this appears to trace the upper envelope of of our estimates and would be consistent with these stars having an angle of inclination close to $90^{\circ}$. Hence assuming that all B-type supergiants undergo the same evolution of rotational velocity $\left(v_{\text {rot }}\right)$ with effective temperature our best estimate for that relationship is given by:

$v_{\text {rot }}=3.4+2.4 \times 10^{-3} T_{\text {eff }}$.

It is emphasised that this relationship should be treated with considerable caution for several reasons. Firstly our sample is relatively small with only 24 targets. Additionally it is unrealistic to assume that every B-type supergiant will follow the same evolutionary history as this will depend on, for example, whether it is evolving on a blue loop having been a red supergiant and whether it is or was part of a binary system. Indeed the relatively tight relationship between projected rotational velocity and effective temperature (excluding Sk 191) in Fig. 2 is surprising. This is because a spread of estimates would be expected due to both the random inclination of rotational axes and to variations in the initial rotational velocity. For example, targets with low $v \sin i$ estimates would be expected covering the full range of effective temperatures as these would have evolved from main sequence O-type stars with low rotational velocities.

An explanation may lie in the biases in our observation samples. For example, two supergiants were excluded from the analysis as it was not possible to identify zeros in their Fourier Transforms, which could be due to them having intrinsically low projected rotational velocities. Hence our results could be 
biased to larger estimates, although as our initial sample size was twenty six targets any bias would be relatively small.

For each part of our sample, the main criteria for target selection was as follows. For the Galactic supergiants, there was a large choice of possible targets and the final selection aimed to cover a range of spectral type for both Ia and Ib luminosity classes. As such there is no clear source of bias in our selection. The VLT spectroscopy was obtained principally to investigate properties of the stellar winds, whilst the AAT targets were chosen to cover a range of photospheric N/C abundances ratios (based on the analysis of McErlean et al. 1999) but with a bias to brighter targets for ease of observation. Hence both SMC samples may be biased to the highest luminosity objects and indeed only contain two objects with luminosity class Iab or Ib. Such samples might be expected to have intrinsically small rotational velocities due to their strong winds. However this would not explain either the lack of low $v \sin i$ objects at the earliest spectra-types or the small scatter in the estimates.

However given all the caveats, discussed above the relationship in Eq. (1) has some merit in indicating the magnitude of the rotational velocities that should be found in evolutionary models of blue supergiants.

\subsection{Comparison with evolutionary predictions}

Previously, several authors have estimated projected rotational velocities and compared these with the predictions of stellar evolutionary calculations. For the A-type supergiants (Venn 1999), there appears to be reasonable agreement. However for O-type dwarfs (Bouret et al. 2003), O-type supergiants (Hiller et al. 2003; Crowther et al. 2002), B-type giants (Lennon et al. 2003) and B-type supegiants (Trundle et al. 2004), the observed values of $v \sin i$ appear to be smaller than those implied by stellar evolutionary calculations. However this comparison is complicated by comparing observed projected rotational velocities with predicted rotational velocities. Additionally it has normally been assumed that rotation was the dominant mechanism in the line broadening (with for example macroturbulence not being included) leading in effect to upper limits for the projected rotational velocities. The advantage of the current estimates are that we have attempted to distinguish between the different broadening mechanisms and thereby have been able to estimate the actual values of projected rotational velocities. In turn, this has allowed us to deduce a relationship between the mean rotational velocity and effective temperature.

In Fig. 3 we compare our results with those predicted by evolutionary models appropriate to both Galactic (Meynet \& Maeder 2003) and SMC metallicities (Meynet \& Maeder 2004). All the models illustrated have initial rotational velocities of $300 \mathrm{~km} \mathrm{~s}^{-1}$ and have initial masses in the range of 20 to $40 M_{\odot}$. As the initial rotational velocities of our targets are unknown (and indeed likely to vary from target to target), this comparison should be taken as illustrative. However all our SMC targets show nuclearsynthetically processed material in their atmospheres and if rotation was indeed the main mechanism for this enrichment, it would appear that their initial rotational velocities must have been relatively large. Also shown are the measured projected rotational velocities and our estimate of the mean rotational velocity as a function of effective temperature taken from Eq. (1). For the Galactic supergiants, our results are in reasonable agreement with the predictions for the $40 M_{\odot}$ model but may be slightly lower than those for the $20 M_{\odot}$. However given the uncertainty in the intitial projected rotational velocities and the relatively small size of our sample, the agreement must be considered encouraging. The evolutionary models appropriate to our SMC sample predict larger rotational velocities - for example at $\log T_{\text {eff }}=4.4 \mathrm{dex}$, the projected rotational velocities for the $40 M_{\odot}$ models are approximately 150 and $70 \mathrm{~km} \mathrm{~s}^{-1}$ at SMC and Galactic metallicities respectively. Although the projected rotational velocity of Sk 191 is consistent with these predictions, those for most of the other SMC targets appear to be too small. This is confirmed by the discrepancy between the theoretical predictions and our estimate of the rotational velocity at early B-spectral types $\left(4.45 \lessgtr \log T_{\text {eff }} \lessgtr 4.25\right.$ ), although the agreement may be better at later spectral types. We note that tests undertaken by Simón-Díaz \& Herrero (2006) indicate that for relatively large macroturbulences and small projected rotational velocities, the FT methodology may overestimate the latter. However allowing for this effect would increase the discrepancy between theory and observation for the SMC dataset.

A comparison is also possible with the models of Heger \& Langer (2000). Unfortunately they do not explicitly tabulate rotational velocities as their models evolve from the main sequence. However their calculations imply that massive stars should evolve to higher effective temperature after their red supergiant stage (the so called "blue loops"). Heger \& Langer predict that such objects should have typical rotational velocities of $50 \mathrm{~km} \mathrm{~s}^{-1}$ and that their maximum effective temperature should be approximately $15000 \mathrm{~K}$. For the late B-type objects, our estimated rotational velocities are smaller but compatible with these predictions.

The theoretical predictions for objects evolving from the main sequence also imply that the amount of processed material mixed to the surface should depend on the initial stellar rotational velocity. For example Maeder \& Meynet (2001) considered $20 M_{\odot}$ evolutionary models with an SMC metallicity and initial rotational velocities ranging from 0 to $400 \mathrm{~km} \mathrm{~s}^{-1}$. At the end of the hydrogen burning phase, these models showed changes in their N/C abundance ratio, which were negligible for the non-rotating model but increased to a factor of approximately six for the fastest rotating model. More recently Meynet \& Maeder (2004) have discussed models with different initial metallicities. For models with significant rotational velocities, they find increases of approximately a factor of ten in the nitrogen abundances as the stars evolve into blue supergiants. Additionally Howarth \& Smith (2001) have discussed the projected rotational velocities for ON and O-type main sequence stars. The cumulative probability function of the former implied that they were rotating more quickly with a Kolmogorov-Smirnov test indicating that the differences were statistically significant. Hence for these O-type stars (that will evolve into B-type supergiants), such a correlation between nitrogen abundances and rotational velocities appears to be present. 


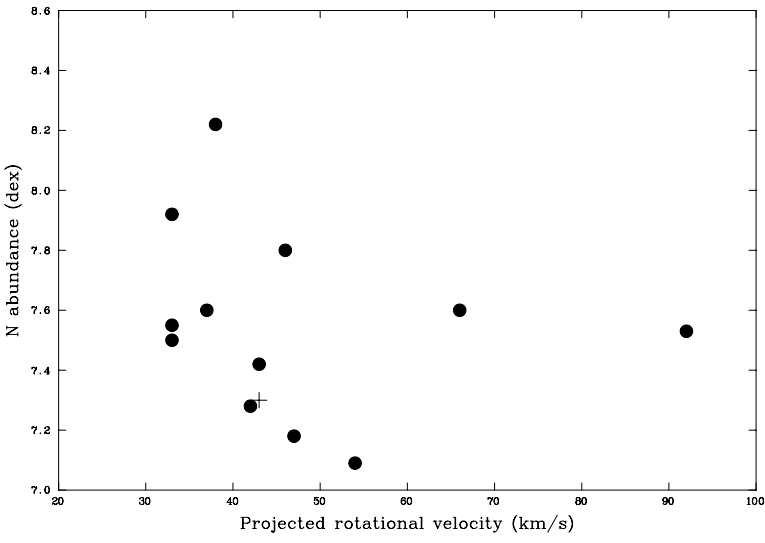

Fig. 4. Projected rotational velocities plotted against nitrogen abundances for the SMC targets. The cross (+) is for the target, AzV 487, for which only an upper limit for the nitrogen abundance could be estimated.

We have searched for such a correlation in our SMC sample (we do not have reliable nitrogen abundances for our Galactic targets) by plotting projected rotational velocities against nitrogen abundances in Fig. 4. The latter have been taken directly from Dufton et al. (2005) and Trundle et al. (2004), although as discussed in Dufton et al. (2005), for two stars in common there was a difference of approximately 0.15 dex in the nitrogen abundance estimates. Tests showed that allowing for this offset made no substantial difference and hence it has not been included in the figure. No positive correlation of nitrogen abundance with projected rotational velocity is observed. Indeed there is a suggestion that our most nitrogen enriched targets have the lowest values of $v \sin i$. However our sample size is relatively small and hence we cannot preclude the possibilty that a correlation has been masked by different angles of inclination and differences in spectral type.

Although our targets normally have relatively small rotational velocities, there is one exception in the SMC supergiant Sk 191. We believe that the result for this star is robust as it is based on two different spectroscopic datasets and is supported by analyses using both the GOF and FT methodologies. Additionally whilst the metal line profiles of the other supergiants appear to be approximately Gaussian, those of Sk 191 have a profile closer to that characterising rotation (see, for example, Gray 1992). However the estimated atmospheric parameters and chemical composition of Sk 191 are consistent with those of the other targets in our SMC sample and hence we are unable to explain the cause of this enhanced rotation.

Hence we would appear to have a situation where effectively all our SMC supergiants have processed material at their surface with most of them having relatively small rotational velocities. Recently, consideration has been given to the effects of magnetic fields (Maeder \& Meynet 2003, 2004, 2005). These would appear to suppress mixing of nucleosynthetically processed material to the stellar surface. However when magnetic and thermal instabilities are also considered, these favour the chemical transport of elements but also lead to larger rotational velocities during the main sequence stage of, for example, a $15 M_{\odot}$ model considered by Maeder \& Meynet (2005).
Hence although magnetic fields may influence the presence of nucleosynthetically processed material at the surface of B-type supergiants, current models do not appear to explain the relatively low rotational velocity estimates found here.

\section{Conclusions}

Our principle conclusions are as follows:

1. We have compared two methodologies for investigating the additional line broadening in spectra of B-type supergiants. We find that Fourier Transforms of the absorption line profiles yield the more reliable estimates of the projected rotational velocity, whilst a "goodness-of-fit" methodology can be used to estimate macroturbulence.

2. We have analysed AAT/UCLES and VLT/UVES spectroscopy of 13 SMC supergiants to estimate the relative contributions of macroturbulence and rotation to the line broadening. These have been supplemented with a reanalysis of spectra of 11 Galactic supergiants.

3. The estimates of the macroturbulence in the SMC and Galactic supergiants show no significant differences. Additionally both datasets show a similar behaviour of the macroturbulence increasing with effective temperature.

4. With the exception of Sk 191, our estimates of the projected rotational velocities show no significant differences between the Galactic and SMC targets, although the latter may be systematically larger by $5-10 \mathrm{~km} \mathrm{~s}^{-1}$. As for the macroturbulence, the estimates of projected rotational velocity increase with effective temperature.

5. Assuming a random orientation of the axis of rotation our results are consistent with a linear behaviour between rotational velocity and effective temperature with value of $v_{\text {rot }}$ of 60 and $30 \mathrm{~km} \mathrm{~s}^{-1}$ at effective temperatures of $28000 \mathrm{~K}$ and 12000 respectively.

6. For our SMC sample, we find no positive correlation between nitrogen abundances and projected rotational velocity as might be expected from stellar evolutionary calculations.

7. Sk 191 has a macroturbulence consistent with its spectral type but an anomalously large projected rotational velocity of $92 \mathrm{~km} \mathrm{~s}^{-1}$.

Acknowledgements. We are grateful to the staff of the AngloAustralian Telescope and the the European Southern Observatory for their assistance and for financial support from the UK Particle Physics and Astronomy Research Council. We would like to thank Prof. I.D. Howarth for useful comments and especially for alerting us to possible observational biases. S.S. and C.T. are grateful for the support of the Spanish Ministerio de Educación y Ciencia through the project AYA2004-08271-C02-01.

\section{References}

Bouret, J.-C., Lanz, T., Hillier, D. J., et al. 2003, ApJ, 595, 1182

Carroll, J. A. 1933a, MNRAS, 93, 478

Carroll, J. A. 1933b, MNRAS, 93, 680

Crowther, P. A., Hillier, D. J., Evans, C. J., et al. 2002, ApJ, 579, 774 
Crowther, P. A., Lennon, D. J., \& Walborn, N. R. 2005, A\&A, submitted

Dufton, P. L., Ryans, R. S. I., Trundle, C., et al. 2005, A\&A, 434, 1125 Ebbets, D. 1979, ApJ, 227, 510

Gray, D. F. 1973, ApJ, 184, 461

Gray, D. F. 1975, ApJ, 202, 184

Gray, D. F. 1992, The observation and analysis of stellar photospheres (Cambridge: Cambridge Univ. Press)

Heger, A., \& Langer, N. 2000, ApJ, 544, 1016

Hillier, D. J., Lanz, T., Heap, S. R., et al. 2003, ApJ, 496, 407

Howarth, I. D., \& Smith, K. C. 2001, MNRAS, 327, 353

Howarth, I. D., Siebert, K. W., Hussain, G. A. J., \& Prinja, R. A. 1997, MNRAS, 284, 265

Hubeny, I. 1988, Computer Phys. Comm., 52, 103

Hubeny, I., \& Lanz, T. 1995, ApJ, 439, 875

Lennon, D. J., Dufton, P. L., \& Crowley, C. 2003, A\&A, 398, 455

Maeder, A., \& Meynet, G. 2001, A\&A, 373, 555

Maeder, A., \& Meynet, G. 2003, A\&A, 411, 543

Maeder, A., \& Meynet, G. 2004, A\&A, 422, 225
Maeder, A., \& Meynet, G. 2005, A\&A, 440, 1041

McErlean, N. D., Lennon, D. J., \& Dufton, P. L. 1998, A\&A, 329, 613

McErlean, N. D., Lennon, D. J., \& Dufton, P. L. 1999, A\&A, 349, 553 Meynet, G., \& Maeder, A. 2000a, A\&A, 361, 159

Meynet, G., \& Maeder, A. 2000b, A\&A, 361, 101

Meynet, G., \& Maeder, A. 2003, A\&A, 404, 975

Meynet, G., \& Maeder, A. 2004, A\&A, 429, 581

Ryans, R. S. I., Dufton, P. L., Rolleston, W. J. R., et al. 2002, MNRAS, 336, 577

Santolaya-Rey, A. E., Puls, J., \& Herrero, A. 1997, A\&A, 323, 488

Schaller, G., Schaerer, D., Meynet, G., \& Maeder, A. 1992, A\&AS, 96, 269

Smith, M. A., \& Gray, D. F. 1976, PASP, 88, 890

Simón-Díaz, S., \& Herrero, A. 2006, A\&A, 448, 351

Simón-Díaz, S., Herrero, A., Esteban, C., \& Najarro, F. 2006, A\&A, 448, 351

Trundle, C., Lennon, D. J., Puls, J., \& Dufton, P. L. 2004, A\&A, 417, 217

Venn, K. A. 1999, ApJ, 518, 405 